\title{
An Adaptive Heuristic Approach for the Multiple Depot Automated Transit Network Problem
}

\author{
Olfa Chebbi ${ }^{(凶)}$, Ezzeddine Fatnassi, and Hadhami Kaabi \\ Institut Supérieur de Gestion de Tunis, Université de Tunis, \\ 41, Rue de la Liberté-Bouchoucha, 2000 Bardo, Tunisia \\ olfaa.chebbi@gmail.com
}

\begin{abstract}
Automated Transit Networks (ATN) are innovative transportation systems where fully driverless vehicles offer an exclusive ondemand transportation service. Within this context of ATN, this study tries to deal with a specific routing problem arising in the context of a ATN's network with a multiple depot topology. More specifically, we present an optimization routing model for automated transit networks which can be used to strategically evaluate depots locations. Our model extends the basic Multi-depot Vehicle Routing Problem (MDVRP). In this paper, the proposed model is tackled using an heuristic approach as the proposed problem is NP-Hard. Experiments are run on a carefully generated instances based on the works from the literature. The numerical results show that the proposed algorithm is competitive as it founds a small gap relative to a lower bound values from the literature.
\end{abstract}

Keywords: Automated transit network - Multi-depot vehicle routing problem $\cdot$ Heuristics $\cdot$ Genetic algorithm

\section{Introduction}

Nowadays, public rapid transit systems provide an interesting way for reducing the distinctive negative impact of transportation tools in urban areas. In fact, public rapid transit systems help to improve the access of lower income groups in societies to transportation tools as well as reducing the environmental impact of urban mobilities. Public rapid transit systems consists of light rapid transit (LRT), bus rapid transit (BRT), Automated Transit Networks (ATN), metro, commuters rail and so on. Recently, several models has been put forward to justify the operational, tactical and strategic implementation of rapid transit systems. In this paper, we focus on the implementation of ATN. We extend the operational model of Mrad and Hidri [10] which is used as a base of our operational ATN model.

In the operational model of Mrad and Hidri [10], the optimized variables are the energy consumption, the objective function is the minimization of total 
energy consumption of ATN. The ATN'network is assumed to have a single uncapacitated depot [7]. We extend this model to account for a multiple depot topology network. We introduce also a maximum allowable distance constraint related to the electric battery capacity of the ATN vehicles. We study the effect of these constraints on the operational level for the multiple depot topology ATN'network. In spite of its relative complexity, the proposed operational model could be solved heuristically based on approximate methods which could yields some analytical insight on the structure of its optimal solutions. In particular, we found that introducing multiple depots topology helps to reduce the total service time for rapid transit users. Also, the proposed heuristic approach was proven to found good quality solutions in a fast computational time.

The remainder of this paper is as follows: Sect. 2 presents the ATN system and its related literature review which motivates our work. Section 3 presents the optimization model. Our proposed heuristic approach is introduced in Sect. 4 . Section 5 provides numerical results analysis of our approach. Conclusions are reported in Sect. 6 .

\section{The Automated Transit Networks}

ATN (also called Personal Rapid Transit (PRT)) consists mainly on a set of small automated driverless electrical vehicles running on a set of exclusive guideways. ATN is implemented to provide an interesting mode of urban transportation service which could address the need of urban mobility based on specific settings. Table 1 provides an overview of the several needs related to urban mobility and how could ATN satisfy them. In the literature, there is a general consensus that the key characteristics of ATN includes [2]: (i) Fully automated vehicles; (ii) Small and dedicated guideways; (iii) On-demand, origin-to-destination service; (iv) Off-line stations; and (v) A network or system of fully connected guideways.

Table 1. ATN main features

\begin{tabular}{l|l}
\hline Need & ATN feature \\
\hline Provide faster service & Non-stop, on-demand service \\
\hline Reduce congestion & $\begin{array}{l}\text { Faster and personalized service to } \\
\text { attract private automobile users }\end{array}$ \\
\hline Reduce pollution & \begin{tabular}{l} 
Electric vehicles \\
\hline Reduce energy use
\end{tabular} \\
$\begin{array}{l}\text { On-demand and Non-stop } \\
\text { transportation service to eliminate } \\
\text { empty vehicle movements }\end{array}$ \\
\hline
\end{tabular}




\subsection{Literature Review}

ATN as a conceptual mode of public rapid transit systems has a history of over 60 years. Since, its first introduction in 1953 [2], it was studied by governments, universities, research organization and so on. Literature of ATN includes several books, scholar papers and technical reports. These studies proposed to treat several features related to ATN such as technical and operational analysis, system design, environmental impact, cost performance and so on.

A literature review published in 2005 [5] states that there is more than 200 research papers related to ATN. More recently, several operational and strategic optimization studies related to ATN were published such as simulation [7], energy minimization [10], total traveled distance [6,8], optimized operational planning [4] and so on. However and from our literature review, many optimization routing models related to ATN considered a single depot network topology $[4,6,10]$.

Consequently, it becomes of a high interest to study optimization routing problem related to ATN based on a multiple network topology. Therefore in the next section, we extends the single depot based optimization model of Mrad and Hidri [10] to propose a multiple depot optimization model which would aim at reducing the total travel time of ATN vehicles while serving a set of known static deterministic list of passengers travels.

\section{The Optimization Model}

In this section, we present the multiple depots ATN optimization model which extends the works of Mrad and Hidri [10]. We first start by presenting the set of assumptions related to our model. Then, we give a graph based model. Finally, we present the complexity of our problem.

\subsection{The Set of Assumptions}

Let suppose that we have a ATN $N$ with a finite number of stations $M$. $N$ satisfies connectivity constraints. Therefore, a ATN vehicle could travel between any pairs of stations in $N$. We suppose that $N$ has a set of depots $\kappa=\left\{d_{1}, d_{2}, d_{3}, \ldots . d_{k}\right\}$ where $k$ represents the number of depot in $N$. In each depot, there exists an unlimited number of ATN vehicles. The exact number of vehicles needed from each depot is considered as a decision variable. Each vehicle has a limited battery capacity denoted $B$. We supposed to have a static pre-deterministic list of trips to serve denoted $T .|T|=n$. Each trip $i$ is identified by a quadruplet:

(i) a depart time $D t_{i}$,

(ii) a depart station $D s_{i}$

(iii) an arrival time $A t_{i}$ and

(iv) an arrival station $A s_{i}$

Finally, let $S P$ be a matrix cost which defines the shortest time travel path between each pair of stations. 


\subsection{Graph Based Formulation}

Our problem has an objective to find a set of least cost roads starting and ending at one of the depots in $N$ which minimizes the total travel time of the ATN vehicle while serving each trip exactly once. To model our problem, let us define $G=\{V, E\}$ where $V$ is a set of nodes and $E$ is a set of edges. Each trip $i$ is represented by a node in $V$. Also, each depot $d_{i}$ is represented by two nodes $s_{i}$ and $t_{i}$. Also, we have $n$ trips and $k$ depots. The cardinality of $V$ is equal to $n+2 k . V^{*}=V \backslash\left\{s_{1}, s_{2}, \ldots s_{k}, t_{1}, t_{2}, \ldots, t_{k}\right\}$. As for the set of edge $E$, it will be defined as following:

- For each pair of nodes $i$ and $j \in V^{*}$, we add an edge $(i, j)$ to $E$ if $A t_{i}+$ $S P_{\left(A s_{i}, D s_{j}\right)} \leq D t_{j}$. The edge has a cost $c_{i j}$, representing the total time needed to move from arrival station $A s_{i}$ of trip $i$ to depart station $D s_{j}$ of trip $j$.

- For each node $i$ and each depot $k$, we add an edge $(k, i)$. This edge has a cost $c_{k i}$ which is equal to total traveled time to reach the depart station $D s_{i}$ of trip $i$, from the depot $k$.

- For each node $i$ and each depot $k$, we add an edge $(i, k)$. This edge has as a cost the total travel time needed to move from the arrival station $A s_{i}$ of trip $i$ to the depot $k$.

Let us also denote $E^{*}=E\{(i, j)$ where $i \in \kappa$ or $j \in \kappa\}$.

\subsection{The Complexity of Our Problem}

Starting from our graph modeling of the problem, we could note that it extends the asymmetric distance constrained vehicle routing problem (ADCVRP) [1]. Our problem is asymmetric as the cost of edge $(i, j) \neq(j, i)$. The DCVRP is a vehicle routing problem where each road is subject to total distance, time or cost constraints. The ADCVRP is not well studied in the literature. In fact and as Almoustafa et al. state [1], only two papers studied this problem [1]. The work related to ADCVRP are based on a single depot topology. Therefore, our proposed ATN problem could be considered as an extension to the ADCVRP by adding multiple depots to its basic version. Thus, it represents an interesting worth to study extension to the works in the literature. The ADCVRP is proven to be an NP-Hard problem [8]. Consequently, our proposed extension to the ADCVRP is an NP-Hard problem. In the next section, we present details of our solution approach proposed to solve our problem.

\section{Genetic Algorithm Approach}

As mentioned earlier, the proposed multiple depot ATN routing problem is an NP-Hard optimization problem which has its own difficulties to solve. Consequently, this paper presents an heuristic approach based on the implementation of genetic algorithm (GA) to solve the proposed optimization problem. GA presents a good solution approach for the proposed ATN problem as it could discover many different zones in the search space [4]. Consequently, it could reach 


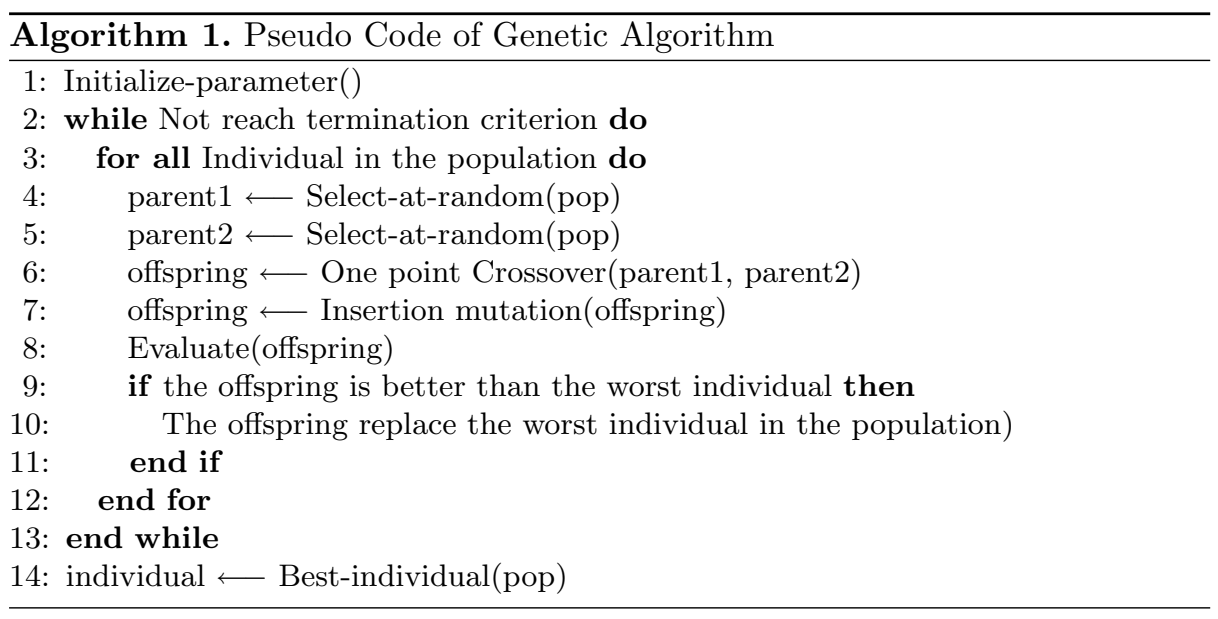

a good quality solution in a fast computational time. A high level overview of our GA is presented in Algorithm 1.

The choice of developing $\mathrm{GA}^{1}$ for this problem is motivated by the fact that large number of studies adopted this solution approach to solve routing problems. One could note for instance [9].

Similarly and starting from a population of individuals, a GA applies genetic operators like crossover and mutation in each iteration in order to generate new offsprings. Consequently, the key issue to successfully develop GA is to select the appropriate genetic operators and solution representation.

In the next subsections, we focus more closely on the proposed GA. We first describe the individual'representation and evaluation function. Then, we discuss the implemented genetic operators and the parameters used therein.

\subsection{Solution Representation and Evaluation Function}

In our GA, a solution is represented using a vector of trips to perform. In this vector, each trip is represented by a single gene only once. Therefore, each solution is in a form of a permutation of trips. As for the evaluation function, we adapt the split function of Prins [11] to our context. More specifically and starting from a permutation, the split function constructs an auxiliary graph where each node represents a trip in addition to a node representing the different depots in the ATN'network. Each edge in the auxiliary graph represents a feasible road based in the permutation at hand. Next, the algorithm uses the shortest path in the auxiliary graph to find the related set of roads. Thus, we obtain the set of roads starting and ending at one of the depots in the network covering each trip only once. More details could be found in [11].

\footnotetext{
${ }^{1}$ Non expert readers can for instance refer to [12] for more details about GA.
} 


\subsection{Crossover and Mutation Operators}

After deciding the representation form of the individuals in the GA, two parents are selected randomly according to Algorithm 1 in order to create new offsprings using crossover operator. Our crossover operator applied in our algorithm is the one point crossover. For the first parent, we choose randomly a cut point. The trips that are present before the cut point in the first parent are copied to the offspring. The missing trips in the resulted offspring are copied from the second parent while following their order of appearance. More details could be found in Fig. 1.

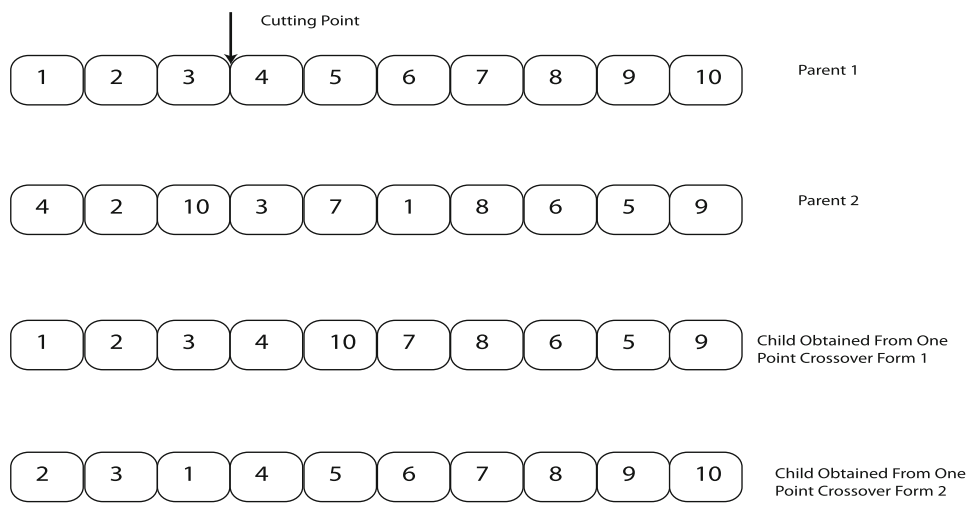

Fig. 1. Example of one point crossover

Also, mutation helps GAs to preserve diversification in the population. In our algorithm, the mutation procedure is applied on the new generated offspring after the crossover operator. In our approach, we use the insertion mutation operator. This operator chooses at random one trip from the permutation and insert it at a random position.

\section{Computational Results}

In this section, we present the computational results related to the proposed GA. The algorithms proposed in this paper were coded in $\mathrm{C}++$ language. The experiments are performed on a $\mathrm{PC}$ with a $3.2 \mathrm{GHZ} \mathrm{CPU}$ and $8 \mathrm{~GB}$ of RAM.

\subsection{Test Instances}

To test our proposed approach, we generated 100 ATN multiple depot instances. The size of the problem (i.e. the number of trips) in our testing bed varies between 10 and 100 trips by a step of 10. For each number of trips, 10 instances 
were generated. To generate the different instances, the ATN's instances generator from the literature of Mrad and Hidri [10] is adapted to our context. To assert the quality of the obtained solutions we used the GAP metric. The GAP is obtained as follows:

$$
\mathrm{GAP}=\left(\frac{(S O L-L B)}{L B}\right) \times 100
$$

We should note that $S O L$ is the solution of $L B$ represents the linear relaxation of the valid mathematical formulation presented in the literature [1]. The mathematical models related to the linear relaxation were implemented using the IBM ILOG CPLEX Optimizer 12.2.

\subsection{Result of the Genetic Algorithm}

As for the parameter tuning, we used a specific method from the literature to effectively tune our proposed GA [3]. Based on this method, we found the following parameters: (i) Number of generations:800, (ii) population size:20; (iii) crossover rate:0.9 and (iv) mutation rate: 0.3 . Table 2 presents the results of our approach. It should be noted the good quality of our proposed GA as we found an average GAP of $2.859 \%$ in $0.231 \mathrm{~s}$.

We should note also that the average GAP grows steadily. The maximum GAP was equal to $6.435 \%$ which is still represents good quality results. As for the average time, our algorithm proved to be very effective as the average computational time was still below $1 \mathrm{~s}$. These results comfort our choice in the selection of a GA for solving our hard combinatorial optimization problem related to ATN. These results are encouraging in term of problem solvability.

Table 2. The Obtained Results

\begin{tabular}{l|l|l}
\hline Number of travels & Average GAP $\%$ & Average time in seconds \\
\hline 10 & 0 & 0.833 \\
\hline 20 & 0.575 & 0.039 \\
\hline 30 & 0.438 & 0.485 \\
\hline 40 & 0.832 & 0.063 \\
\hline 60 & 1.771 & 0.082 \\
\hline 70 & 3.327 & 0.103 \\
\hline 90 & 4.263 & 0.122 \\
\hline 100 & 4.628 & 0.148 \\
\hline Average & 6.327 & 0.191 \\
\hline & 6.435 & 0.241 \\
\hline
\end{tabular}




\section{Conclusions}

In this paper the Multi-Depot automated transit network problem is evoked and modeled. A genetic algorithm is proposed and implemented to solve it. The proposed algorithm integrates an effective genetic operators and evaluation function for solving the combinatorial optimization problem. The algorithm constructs a set of ATN'vehicles routes starting and ending at any of the proposed depots with minimum routing costs. Computational experiments on a set of carefully generated instances show that the proposed heuristic is very effective. As an extension to this work, a more adapted meta-heuristic approach such as bee colony algorithms, ant colony algorithm could be adapted to our context. Also the inclusion of additional constraints such as mixed fleet with varying maximum allowable distance and multi-compartment vehicles is under investigation.

Open Access. This chapter is distributed under the terms of the Creative Commons Attribution 4.0 International License (http://creativecommons.org/licenses/by/ 4.0/), which permits use, duplication, adaptation, distribution and reproduction in any medium or format, as long as you give appropriate credit to the original author(s) and the source, a link is provided to the Creative Commons license and any changes made are indicated.

The images or other third party material in this chapter are included in the work's Creative Commons license, unless indicated otherwise in the credit line; if such material is not included in the work's Creative Commons license and the respective action is not permitted by statutory regulation, users will need to obtain permission from the license holder to duplicate, adapt or reproduce the material.

\section{References}

1. Almoustafa, S., Hanafi, S., Mladenovi, N.: New exact method for large asymmetric distance-constrained vehicle routing problem. Eur. J. Oper. Res. (2012)

2. Carnegie, J.A., Hoffman, P.S.: Viability of personal rapid transit in New Jersey. Technical report (2007)

3. Chebbi, O., Chaouachi, J.: Effective parameter tuning for genetic algorithm to solve a real world transportation problem. In: 2015 20th International Conference on Methods and Models in Automation and Robotics (MMAR), pp. 370-375. IEEE (2015)

4. Chebbi, O., Chaouachi, J.: Reducing the wasted transportation capacity of personal rapid transit systems: an integrated model and multi-objective optimization approach. Transp. Res. Part E Logistics Transp. Rev. 89, 236-258 (2015)

5. Cottrell, W.D.: Critical review of the personal rapid transit literature. In: Proceedings of the 10th International Conference on Automated People Movers, pp. 1-4, May 2005

6. Fatnassi, E., Chebbi, O., Chaouachi, J.: Discrete honeybee mating optimization algorithm for the routing of battery-operated automated guidance electric vehicles in personal rapid transit systems. Swarm Evol. Comput. 26, 35-49 (2015)

7. Fatnassi, E., Chebbi, O., Siala, J.C.: Two strategies for real time empty vehicle redistribution for the personal rapid transit system. In: 2013 16th International IEEE Conference on Intelligent Transportation Systems (ITSC), pp. 1888-1893. IEEE (2013) 
8. Fatnassi, E., Chebbi, O., Siala, J.C.: Comparison of two mathematical formulations for the offline routing of personal rapid transit system vehicles. In: The International Conference on Methods and Models in Automation and Robotics (2014)

9. Lahyani, R., Khemakhem, M., Semet, F.: Rich vehicle routing problems: from a taxonomy to a definition. Eur. J. Oper. Res. 241(1), 1-14 (2015)

10. Mrad, M., Hidri, L.: Optimal consumed electric energy while sequencing vehicle trips in a personal rapid transit transportation system. Comput. Ind. Eng. 79, 1-9 (2015)

11. Prins, C., Lacomme, P., Prodhon, C.: Order-first split-second methods for vehicle routing problems: a review. Transp. Res. Part C Emerging Technol. 40, 179-200 (2014)

12. Sivanandam, S., Deepa, S.: Introduction to Genetic Algorithms. Springer Science \& Business Media, New York (2007) 